\title{
Prone positioning in severe acute hypoxemic respiratory failure in the emergency ward
}

\author{
Andrea Bellone, Adriano Basile \\ Emergency Ward, Niguarda Hospital, Milan, Italy
}

\begin{abstract}
This paper reports the authors' preliminary experience with three patients affected by severe acute hypoxemic respiratory failure due to bilateral pneumonia placed in a prone position in Emergency Medicine Ward during application of high flow oxygen nasal cannula.
\end{abstract}

\section{Introduction}

It is well known that, compared with the supine position, placing patients in a prone position determines a beneficial effect of tidal volume distribution, in part by reversing the vertical pleural pressure gradient, which becomes more negative in the dorsal pulmonary regions, thus improving the ventilation/perfusion ratio. Prone position also improves resting lung volumes in dorso-caudal regions by reducing the superimposed pressures of both heart and abdominal organs. ${ }^{1-3}$ The final result of prone positioning in most subjects with acute hypoxemic respiratory failure due to severe pulmonary consolidation is to exhibit mild to dramatic improvements in oxygenation.

\section{Case Report}

In the last three months, three patients were admitted to our Emergency Medicine Ward because they were affected by acute

Correspondence: Andrea Bellone, Niguarda Hospital, Piazza Ospedale Maggiore 3, 20162 Milan, Italy.

Tel: +39.02 .64447433 .

Email: andrea.bellone@ospedaleniguarda.it

Key words: Prone positioning; Acute respiratory failure; Pneumonia.

Contributions: the authors contributed equally.

Conflict of interest: the authors declare no potential conflict of interest.

Funding: none.

Received for publication: 26 April 2018.

Revision received: 21 May 2018.

Accepted for publication: 24 May 2018.

This work is licensed under a Creative Commons Attribution 4.0 License (by-nc 4.0).

(C) Copyright A. Bellone and A. Basile, 2018

Licensee PAGEPress, Italy

Emergency Care Journal 2018; 14:7524

doi:10.4081/ecj.2018.7524 respiratory failure due to pneumonia. Their chest computed tomography (CT) reveals pulmonary infiltrates and ground glass opacities. All patients complained dyspnea, cough and presented arterial blood gases with $\mathrm{PaO}_{2} / \mathrm{FiO}_{2}$ ratio $<150$. Figures 1 and 2 show the chest X-ray and chest CT scan of one of the three patients.

We obtained also an early Intensivist evaluation, which excluded endo-tracheal intubation and admission to Intensive Care Unit.

Antibiotics were immediately administrated, saline infusion as needed and patients were submitted to a trial of non-invasive mechanical ventilation. We started with Bi-level Positive Airway Pressure (BiPAP) with a positive end-expiratory pressure (PEEP) of 8 to $12 \mathrm{~cm} \mathrm{H}_{2} \mathrm{O}$, pressure support at 8 to $10 \mathrm{~cm} \mathrm{H}_{2} \mathrm{O}$ and $\mathrm{FiO}_{2}$ at $50 \%$. Every four hours BiPAP was transient interrupted and alternated with two hours of high flow oxygen nasal cannula (HFNC) applied at $\mathrm{FiO}_{2}$ at $50 \%$ with a $60 \mathrm{~L} /$ min flow.

As reported in Table 1 , the most important end points $\left(\mathrm{PaO}_{2} / \mathrm{FiO}_{2}\right.$ ratio and respiratory frequency) did not show any significant improvement in the first 48 hours. For this reason, we placed our patients in a prone position for six hours/day with immediate beneficial effects, both on symptoms and gas exchange. The combination of prone positioning and BiPAP/HFNC improved significantly $\mathrm{PaO}_{2} / \mathrm{FiO}_{2}$ ratio with a reduced respiratory frequency within three-four days. Arterial blood gases data (mean values and range) of patients are reported in Table 1.

We decided to apply HFNC during prone positioning because it was more confortable than BiPAP for a not-intubated patient.

HFNC is a relatively recent method to deliver oxygen in hypoxemic patients. The main advantages of HFNC versus conventional oxygen therapy are: maintenance of warm and humidified gas flow through airways, a good carbon dioxide washout and generation of a slight PEEP.-7

After ten days from admission in our Emergency Medicine Ward, the patient was transferred to Internal medicine ward. On 9, 10 or $11^{\text {th }}$ day from admission a chest computed tomography was repeated, showing a complete or almost complete regression of ground glass opacities and alveolar consolidations of both lungs. Within 10 days from the admission all patients were weaned by ventilators/HFNC and on 14 to $16^{\text {th }}$ day from the admittance in the Emergency ward, patients were discharged from Hospital.

To our knowledge, this approach is the first in a setting of Emergency Medicine Ward, about patients affected by acute hypoxemic respiratory failure due to pneumonia underwent to a prone positioning as an additional therapy when HFNC and BiPAP were ineffective alone. This case highlights the usefulness of prone positioning as a complementary treatment of acute hypoxemic respiratory failure in an Emergency Medical Ward.

\section{Conclusions}

Prone positioning has been used for many years to improve oxygenation in patients who require mechanical ventilator support for management of acute respiratory distress syndrome in 

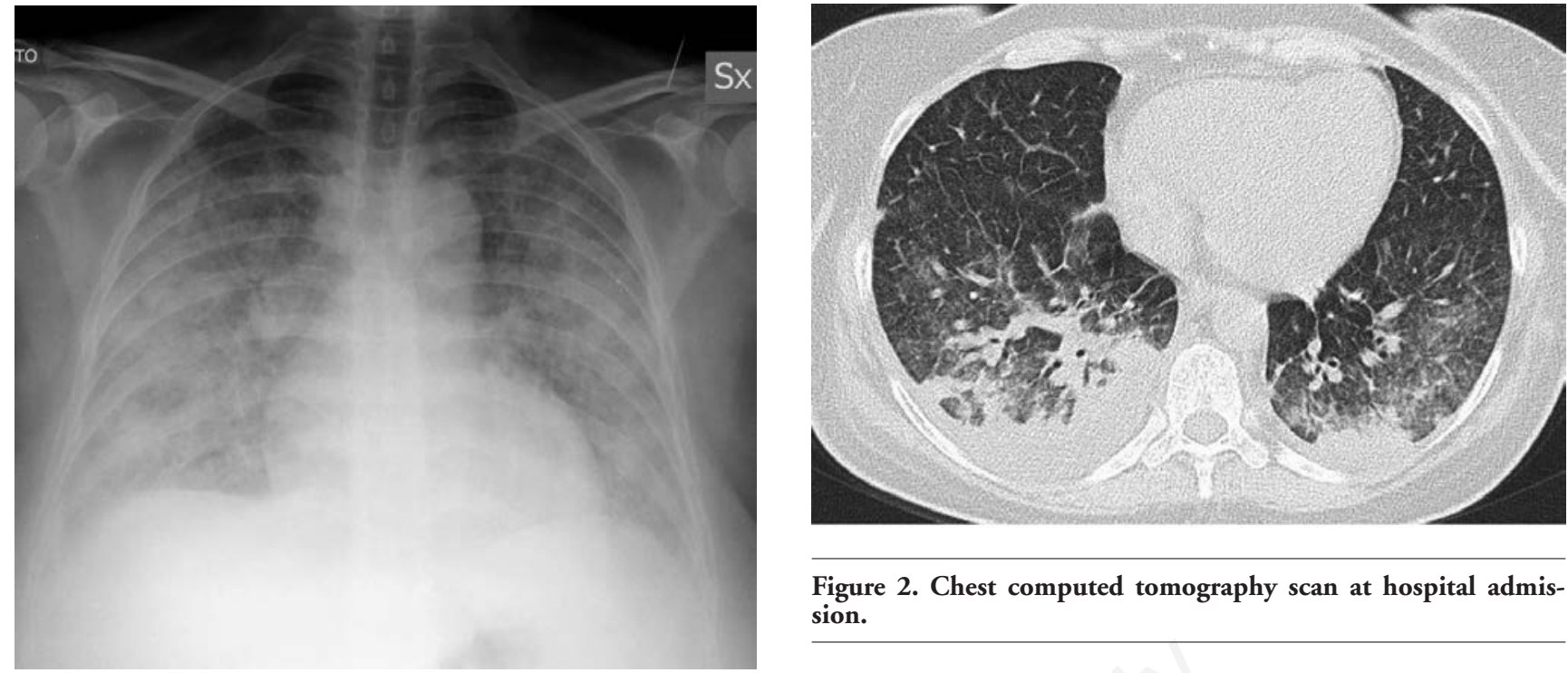

Figure 2. Chest computed tomography scan at hospital admission.

Figure 1. Chest X-ray at hospital admission.

Table 1. Arterial blood gas analysis (mean values and range). $\mathrm{PaO}_{2}, \mathrm{PaCO}_{2}$ : $\mathrm{mmHg}$.

\begin{tabular}{lccccc} 
& Day 1 & Day 2 & Day 3 & Day 6 & Day 9 \\
$\mathrm{PaO}_{2} / \mathrm{FiO}_{2}(\%)$ & $13(107-149)$ & $118(91-137)$ & $275(265-284)$ & $283(261-299)$ & $303(292-319)$ \\
$\mathrm{PaCO}_{2}(\mathrm{mmHg})$ & $28(24-31)$ & $30(27-34)$ & $34(30-39)$ & $37(34-39)$ & $39(36-42)$ \\
$\mathrm{pH}$ & $7.49(7.46-7.51)$ & $7,46(7.45-7.47)$ & $7,41(7.38-7.42)$ & $7,40(7.36-7.43)$ & $7,42(7.38-7.43)$ \\
$\mathrm{HCO}_{3}(\mathrm{mmol} / \mathrm{L})$ & $21(19-23)$ & $21(18-23)$ & $20(17-23)$ & $22(19-24)$ & $23(20-24)$ \\
\hline Respiratory rate (breath/min) & $38(35-39)$ & $33(30-35)$ & $24(22-26)$ & $16(14-17)$ & $16(14-17)$ \\
\hline
\end{tabular}

Intensive Care Units. Our report describes three cases of acute hypoxemic respiratory failure due to bilateral pneumonia submitted to a prone positioning in Emergency Medicine Ward as a complementary treatment of high flow oxygen nasal cannula and BiPAP. Our preliminary study needs an accurate prospective protocol for demonstrating the effectiveness and the beneficial of our hypothesis, but the introduction of prone positioning in the clinical practice of the emergency wards seems, in our opinion, very promising.

This case highlights the usefulness of prone positioning as a complementary treatment of acute hypoxemic respiratory failure due to pneumonia in addition to HFNC and BiPAP in an Emergency Medicine Ward.

\section{References}

1. Abrough F, Ouanes-Besbes L, Elatrous S, Brochard L. The effect of prone positioning in acute distress syndrome or acute lung injury: a meta-analysis: areas of uncertainty and rec- comendations for research. Intens Care Med 2008;34:1002-11.

2. Sud S, Friedrich JO, Taccone P, et al. Prone ventilation reduces mortality in patients with acute respiratory failure and severe hypoxemia: systematic review and meta-analysis. Intens Care Med 2010;35:585-99.

3. Kallet RH. A comprehensive review of prone position in ARDS. Respiratory Care 2015;60:1660-87.

4. Dysart K, Miller TL, Wolfson MR, Shaffer TH. Research in high flow therapy: mechanisms of action. Respir Med 2009;103:1400-5.

5. Sztrymf B, Messika J, Bertrand F, et al. Beneficial effects of humidified high flow nasal oxygen in critical care patients: a prospective pilot study. Intens Care Med 2011;37:1780-6.

6. Frat JP, Thille AV, Mercat A, et al. High-flow oxygen through nasal cannula in acute hypoxemic respiratory failure. $\mathrm{N}$ Engl J Med 2015;372:2185-96.

7. Nishimura M. High-flow nasal cannula oxygen therapy in adults: physiological benefits, indication, clinical benefits, and adverse effects. Respiratory Care 2016;61:529-41. 